\title{
Design of RS and D-Flip-Flop using AIGaAs/GaAs MODFET Technology
}

\author{
Ganesan.V \\ Assistant Professor \\ Sathyabama UniversityChennai-600119. \\ Tamilnadu, India
}

\author{
Shaji.K.S \\ Principal \\ Rajas international Institute of Technology For \\ Women, Nagercoil, Tamilnadu, India
}

\begin{abstract}
This paper enumerates high speed design of RS \& D- flip-flop using AlGaAs/GaAs MODFET. The proposed Flip Flop is having less number of transistors than existing designs. Simulation results show lowest average power and least delay than existing designs. This Flip-Flop having less number of transistors.It can be efficiently used in VLSI ICs. In the verification by simulation, the proposed flip-flops appear to have better speed of operation. It is simple and suitable to SPICE simulation of hybrid digital ICs.
\end{abstract}

\section{Keywords}

Flip-Flop, MODFET, delay, PDP, power consumption.

\section{INTRODUCTION}

Technology scaling of a transistor feature size has provided a remarkable innovation in silicon industry for the past few decades. Designers are striving for semiconductor area, higher speed, low power consumption and reliability due to ever increasing demand and popularity of portable electronics. With the increasing use of mobile devices, consumer electronics markets demand a stringent constraint on reducing the power dissipation. In order to reduce the complexity of circuit design, the digital circuits are designed to be synchronous circuits. The memory elements in a sequential circuit are called flip-flops. A flip-flop has two outputs, one for the normal value and one for the complement value of the stored bit. Binary information can enter into a flip-flop in a variety of ways and gives rise to different types of flip-flops. MODFETs are used in integrated circuits as digital on-off switch. FETs can also be used as amplifiers for large amounts of current using a small voltage as a control signals. Both of these are made possible by the FET's unique current-voltage characteristics. MODFETs are heterojunctions. This means that the semiconductors used have dissimilar band gaps. When a heterojunction is formed, the conduction band and the valence band throughout the material must bend in order to form a continuous level.The Modulation doping field effect transistor has demonstrated characteristics which make it highly suitable for high performance high speed system. In this paper describes high speed RS \& D-flip flops using MODFET technology. In this paper, logic gates are designed using RTL logic, So the number of transistors used for making Flip Flops is very less and the area of the Flip Flops is also reduced. The design for low power issues can't be overcome without precised power prediction and optimization tools. Therefore, the critical need for certain tools to calculate power dissipation during the design to meet the power constraints to ignore the costly redesign effort. Power consumption is affected by many factors, $\mathrm{P}=\alpha \mathrm{Cf}$ [7]

\section{CIRCUIT DESIGN}

\subsection{RS FLIP FLOP}

Please use a 9-point Times Roman font, or other Roman font When using logic gates as building blocks, the fundamental latch is the simple SR latch, where S and R stands for set and reset. And it can be constructed from a pair of cross-coupled NOR gate logic. The stored bit is present on the output marked Q.

While the $\mathrm{S}$ and $\mathrm{R}$ inputs are both low, feedback maintained the $\mathrm{Q}$ and $\mathrm{Q}$ outputs in a constant state, with $\mathrm{Q}$ the complement of Q. If $\mathrm{S}$ (set) is pulsed high while $\mathrm{R}$ (Reset) is held low, then the Q output is forced to high, and stays high when $S$ will returns to low, similarly, if $R$ is pulsed high while $\mathrm{S}$ (set) is held low, then the Q output is forced to low, and stays low when $\mathrm{R}$ returns to low. The logic diagram of SR flip flops is shown in fig. 2 and circuit diagram is shown in fig.3. Clocked RS Flip-flop operates as a standard bistable latch but the outputs are only activated when a logic "1" is applied to its EN input and deactivated by a logic " 0 ". The property of this flip-flop is summarized in its characteristic table where Qn is the logic state of the previous output and $\mathrm{Qn}+1$ is that of the next output and the clock input being at logic 1 for all the $\mathrm{R}$ and $\mathrm{S}$ input combinations. The logic diagram of clocked SR flip flops is shown in fig. 3 and circuit diagram is shown in fig. 4

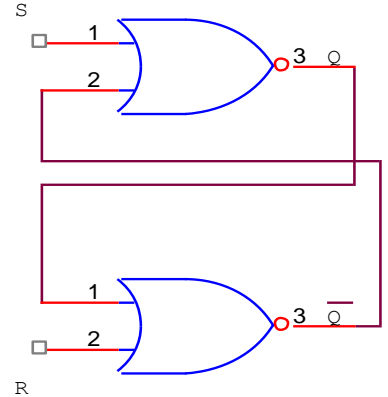

Fig 1: Logic diagram of SR Flip Flops using NOR gate 
Table 1. SR latch operation

\begin{tabular}{|l|l|l|}
\hline S & $\mathbf{R}$ & Action \\
\hline 0 & 0 & No Change \\
\hline 0 & 1 & $\mathrm{Q}=1$ \\
\hline 1 & 0 & $\mathrm{Q}=0$ \\
\hline 1 & 1 & Restricted combination \\
\hline
\end{tabular}

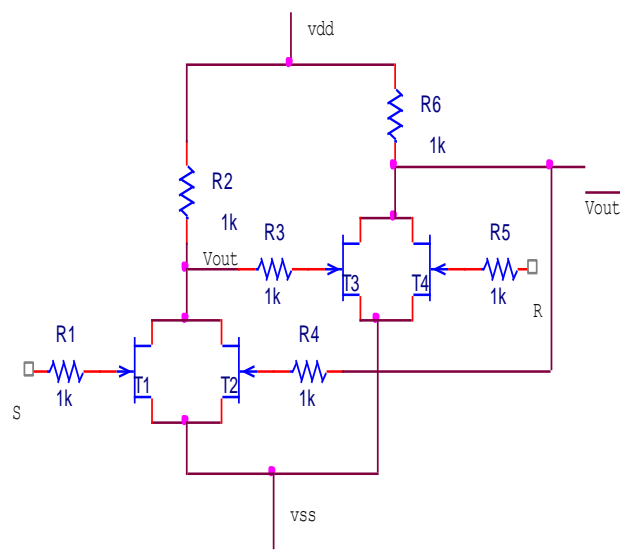

Fig 2: Circuit diagram of SR Flip Flops using NOR gate

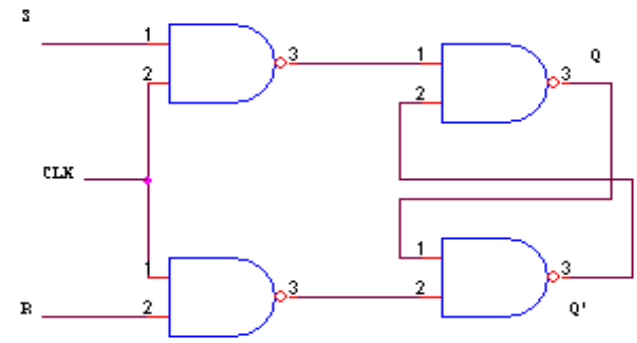

Fig 3: Logic diagram of Clocked SR Flip Flops using NAND gate

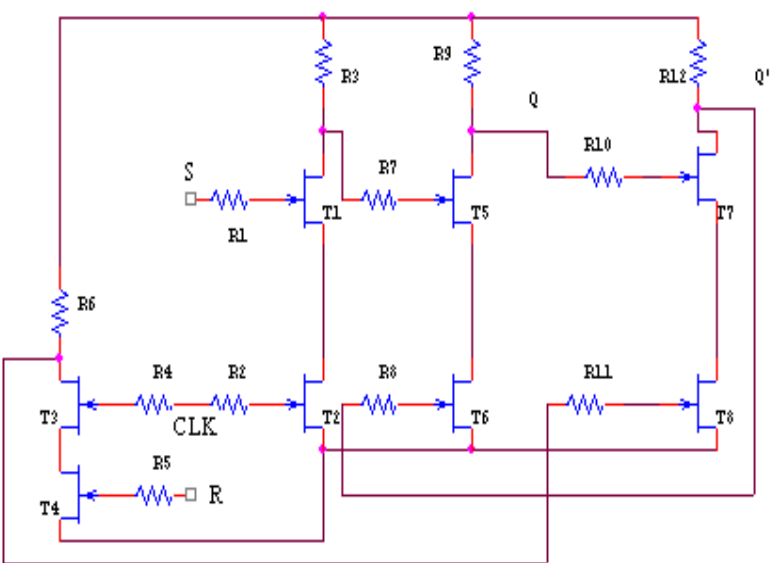

Fig 4: Circuit diagram of Clocked SR Flip Flops using NAND gate

\subsection{FLIP FLOP}

The D flip-flop is shown in Figure 6 is a modification of the clocked SR(Set-Reset) flip-flop. The D input goes directly into the $\mathrm{S}$ input and the complement of the $\mathrm{D}$ input goes to the $\mathrm{R}$ (Reset) input. The $\mathrm{D}$ input is sampled during the occurrence of a clock pulse. If it is 1 (on), the flip-flop is switched to the set state (unless it was already set). If it is 0 (off), the flip-flop switches to the clear state. The logic diagram of D flip flops is shown in fig. 6 and circuit diagram is shown in fig. 7

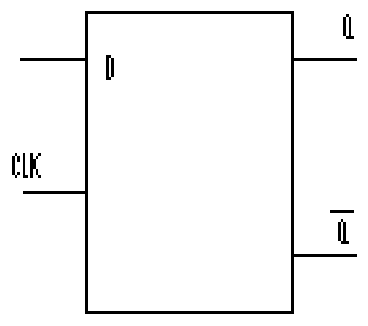

Fig 5: Symbol for an D Flip Flop

Table 2. Truth table for D flip-flop

\begin{tabular}{|l|l|l|}
\hline Clk & D & Q \\
\hline 0 & 0 & 0 \\
\hline 0 & 1 & 1 \\
\hline 1 & 0 & 0 \\
\hline 1 & 1 & 1 \\
\hline
\end{tabular}

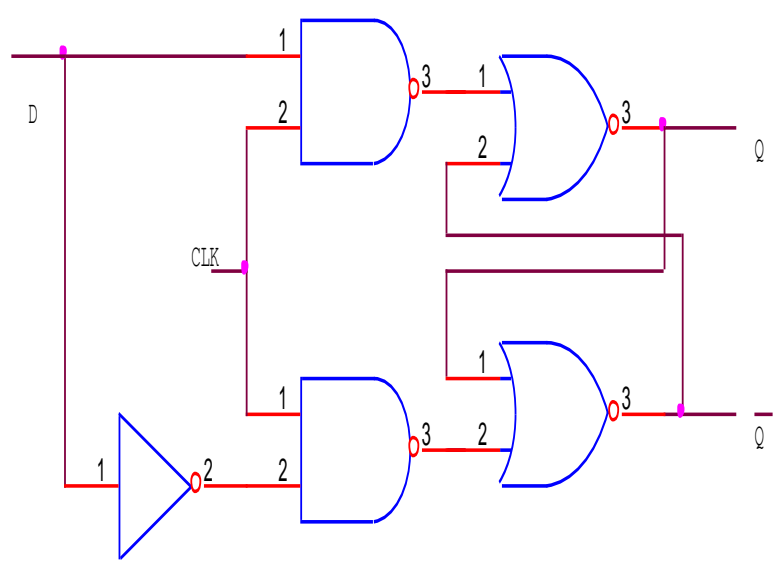

Fig 6: Logic diagram of D Flip Flops 


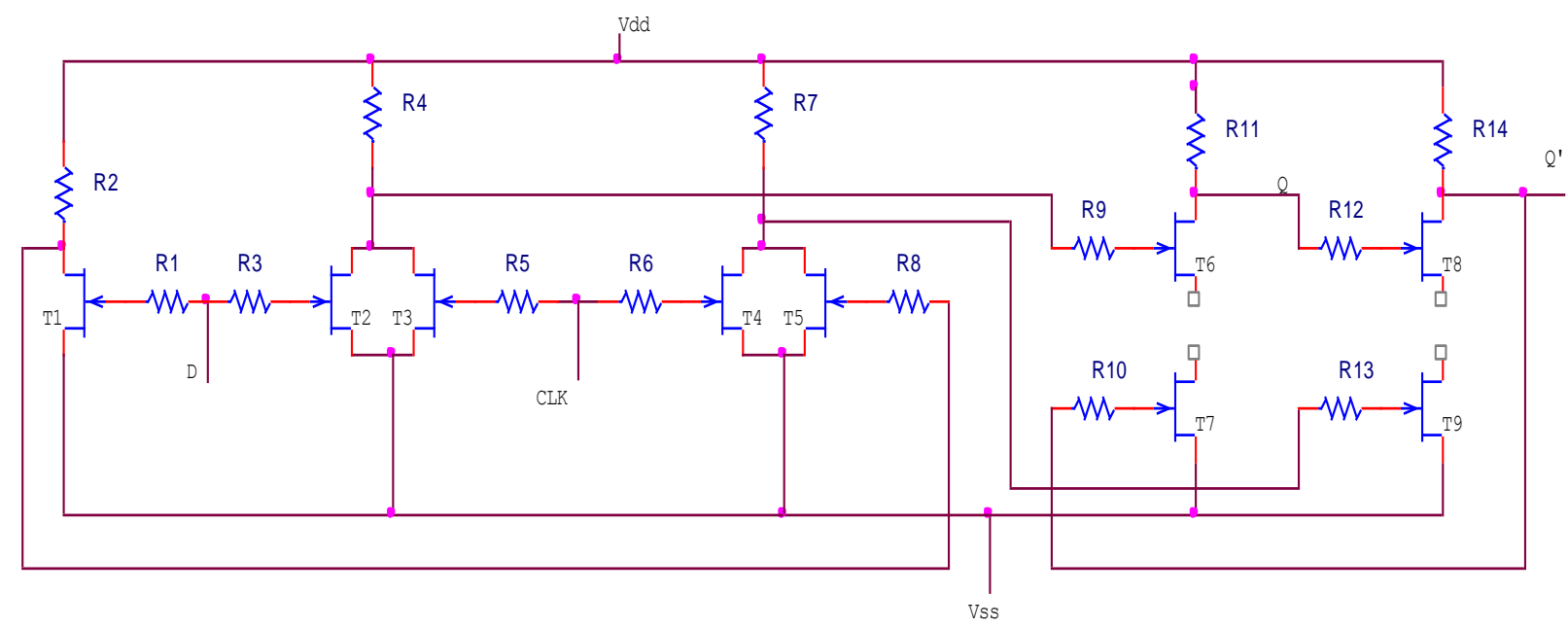

Fig 7: Circuit Diagram of D Flip Flop using MODFET

\section{PERFORMANCE ANALYSIS}

To evaluate the performance of proposed RS and D flip flop using MODFET technology. Simulations are carried out using PSPICE tool in nominal conditions with operating frequency at $1 \mathrm{GHz}$. Transient analysis of the proposed RS and D flip flop using MODFET technology is shown in figure 9 and 10.
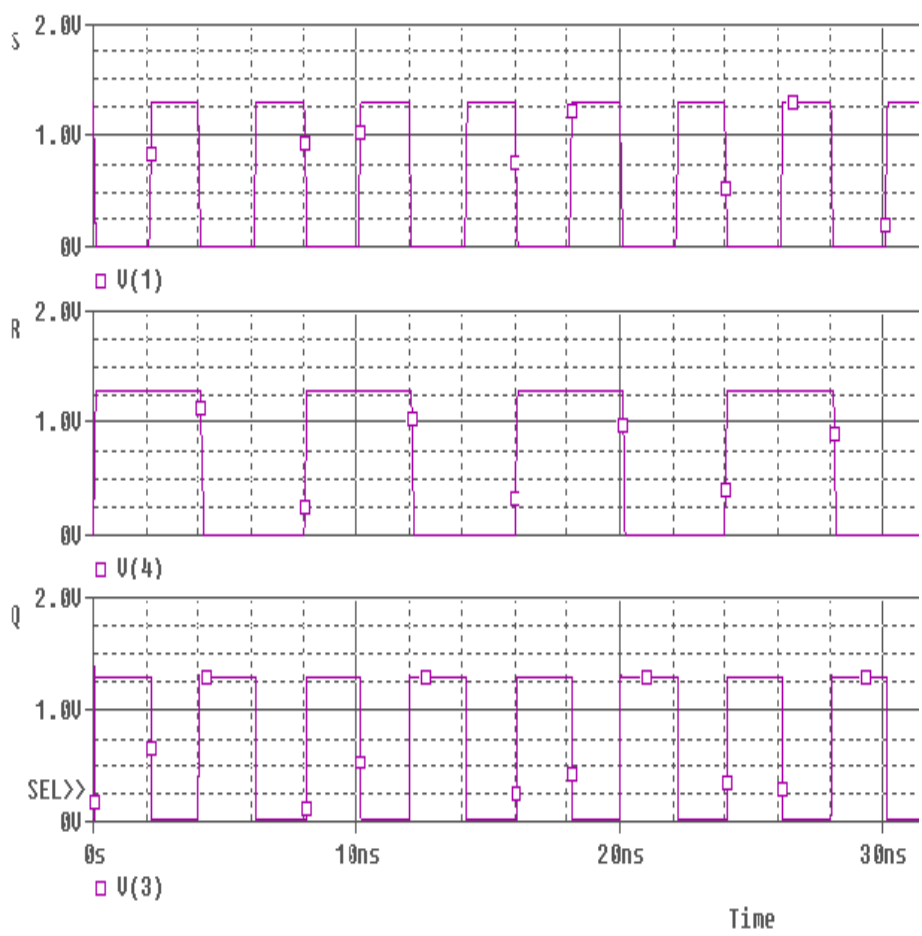

Fig 8: Output waveform for proposed RS Flip Flop

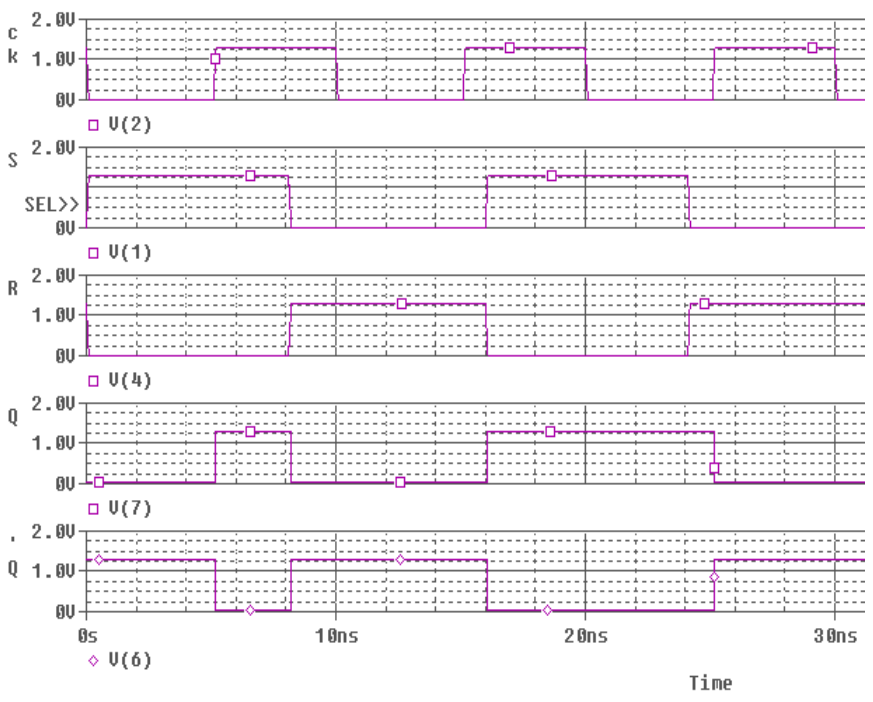

Fig 9: Output waveform for proposed Clociked RS Flip Flop
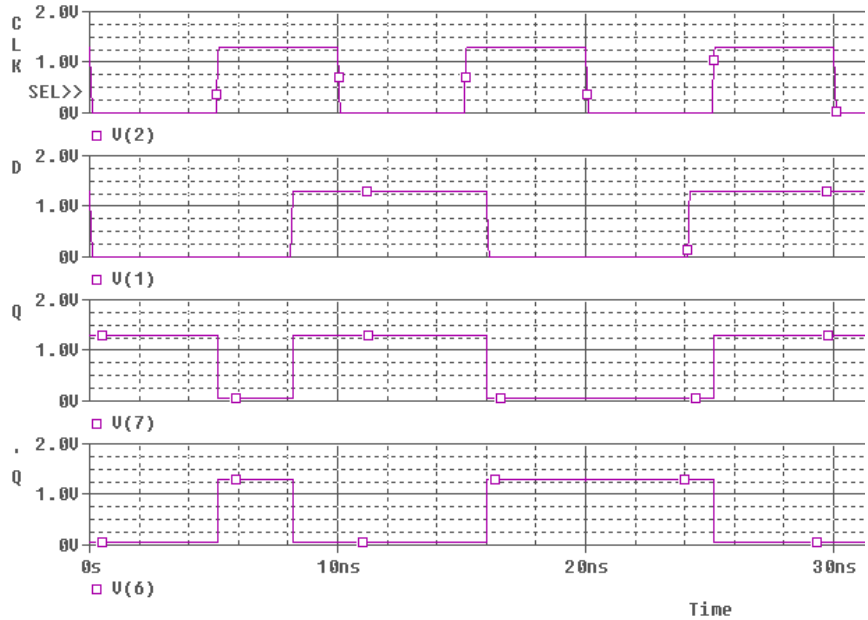

Fig 10: Output waveform for proposed D Flip Flop 


\section{COMPARISON OF MOSFET, AND MODFET D FLIP-FLOPS}

The proposed RS and D flip flop using MODFET is designed and compared with several conventional Flip-Flops. Each Flip-Flop is optimized for power delay product. The proposed flip flop is having lesser number of clocked transistors than the other flip flop. Simulation results for power, delay, PDP and area at nominal conditions for the Flip-Flops are summarized in Table 3. And comparison graph is shown in the fig. $11 \mathrm{a}, 11 \mathrm{~b}, 11 \mathrm{c}$.

Table 2. Comparison of MOSFET, CNTFET and Proposed MODFET D flip flops

\begin{tabular}{|l|c|c|c|}
\hline Device & Delay & Power & PDP \\
& $\left(* \mathbf{1 0}^{-\mathbf{1 2}} \mathbf{S e c}\right)$ & $\left(* \mathbf{1 0}^{-\mathbf{6}} \mathbf{W}\right)$ & $\left(* \mathbf{1 0}^{-\mathbf{1 7}} \mathbf{J}\right)$ \\
\hline Con. DFF & 55 & 4.23 & 23.27 \\
\hline Proposed & 26 & 2.80 & 7.28 \\
\hline
\end{tabular}

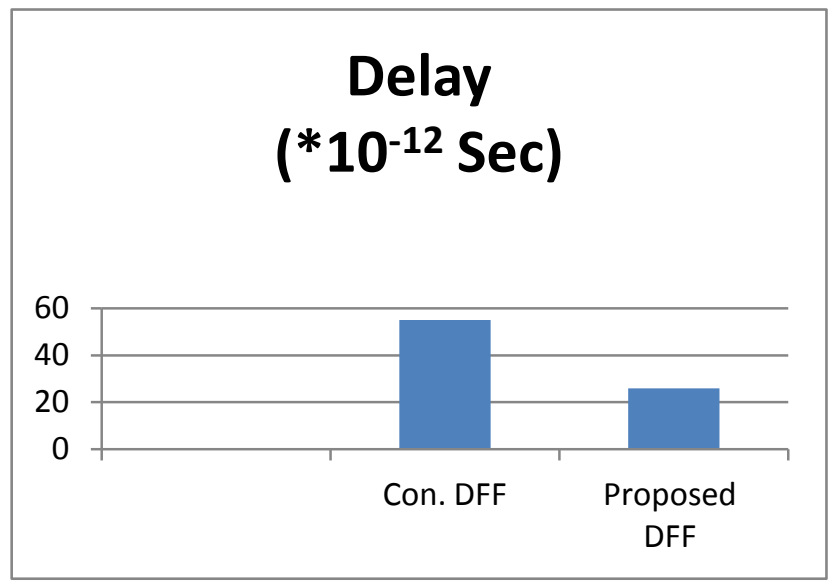

Fig 11a: Comparison of MOSFET and MODFET - Delay

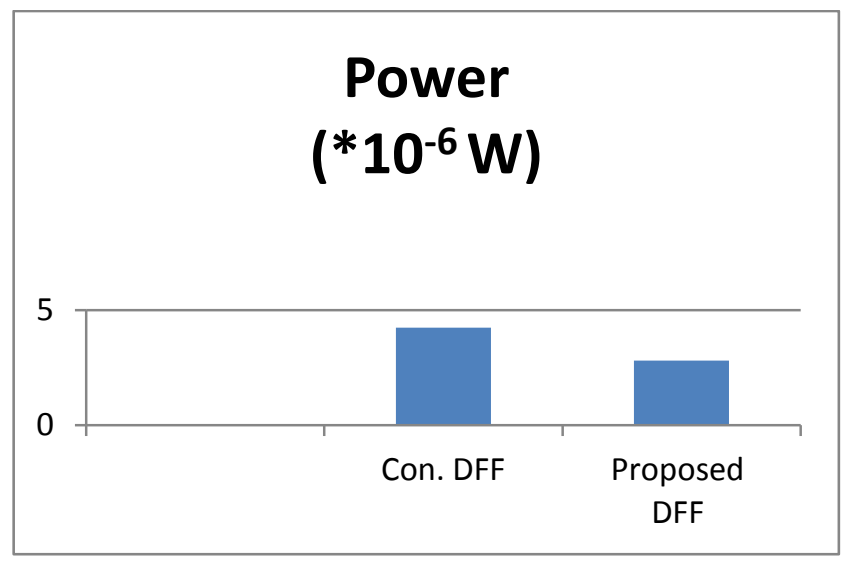

Fig 11b: Comparison of MOSFET and MODFET - Power

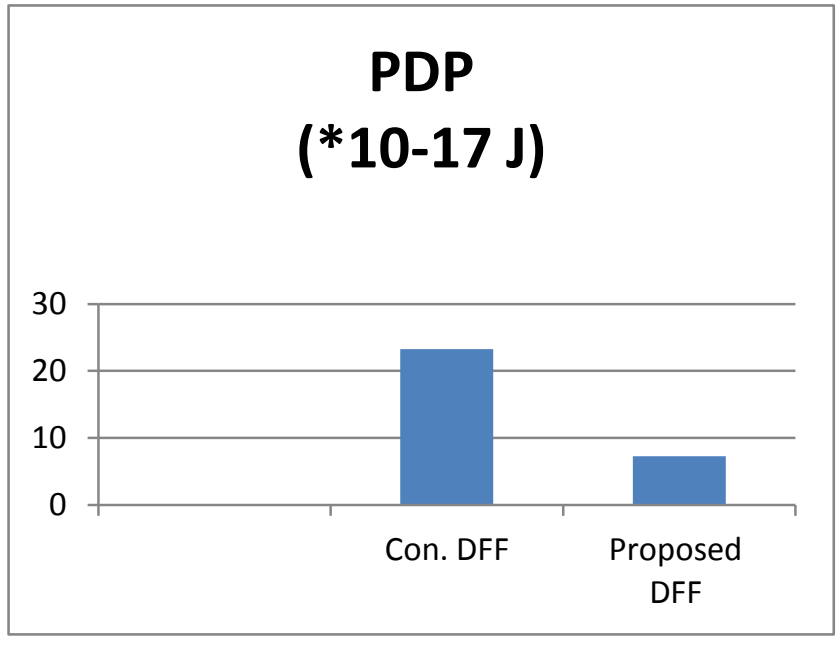

Fig 11c: Comparison of MOSFET and MODFET - PDP

\section{CONCLUSION}

This paper deals with designed of an efficient RS and D flip flop using AlGaAs/GaAs MODFET technologies. The Flip Flop are simulated in PSPICE with operating voltage of $1 \mathrm{~V}$ and operating frequency at $1 \mathrm{GHz}$ in MODFET and compared the performances. MODFET based flip flop have 55\% less power consumption, delay has also decreased and power delay product (PDP) has been reduced $70 \%$ than MOSFET design. The rise time and fall time has also been decreased. The MODFET based flip flop are more efficient in average power, delay, power delay product, rise time and fall time. Hence from performance analysis of an RS and D flip flop using MODFET is more efficient for low power and high performance applications.

\section{ACKNOWLEDGMENTS}

The authors are grateful to the management of Sathyabama University, Chennai, especially to Dr.Jeppiaar, chancellor, Dr.Marie Johnson \& Mrs.Mariazeena Johnson, Directors for providing the necessary facilities for carrying out this research.

\section{REFERENCES}

[1] Imran Ahmed Khan, Danish Shaikh and Mirza Tariq Beg, "2 GHz Low Power Double Edge Triggered flip- flop in 65nm CMOS Technology," IEEE Conference, 2012.

[2] Xiaowen Wang and William H. Robinson, “A Low-Power Double Edge-Triggered Flip-Flop with Transmission Gates and Clock Gating," IEEE Conference, pp 205-208, 2010.

[3] Yu Chien-Cheng,, "Low-Power Double Edge- Triggered Flip-Flop Circuit Design," Third International Conference on Innovative Computing Information and Control (ICICIC'08), IEEE Conference, 2008.

[4 Kandukuru (M); Prakasam(Dt), "Design Approaches for Low power-Low area D flip flop in Nano Technology," International Journal of Electronics Signals and Systems (IJESS) ISSN: 2231- 5969, Vol-2 Iss-1, 2012

[5] Peiyi Zhao, Jason McNeely, Pradeep Golconda and Jianping $\mathrm{Hu}$, "Low Power Design of Double-Edge Triggered Flip-Flop by Reducing the Number of Clocked Transistors," IEEE Conference, 2008. 
[6] Sandeep Sriram, Arun Ramnath, Haiqing, Hojoon Lee and Ken Choi "A Novel Dual Edge Triggered NearThreshold State Retentive Latch Design," IEEE Conference, 2011.

[7] Peiyi Zhao, Jason McNeely, Pradeep Golconda and Jianping $\mathrm{Hu}$, "Low Power Design of Double-Edge Triggered Flip-Flop by Reducing the Number of Clocked Transistors," IEEE Conference, 2008.

[8] M. Pedram, Q. Wu, and X. Wu, "A New Design of Double Edge Triggered Flip-Flops," Proceedings of the Asia and South Pacific Design Automation Conference (ASPDAC), pp. 417-421, 1998.

[9] Troy A. JohnsonP and Ivan S. Kourtev , "A Single Latch, High Speed Double-Edge-triggered flip-flop (DETFF)" IEEE, 2001, in press.

[10] Yu Chien-Cheng, "Design of Low-Power Double EdgeTriggered Flip-Flop Circuit", Second IEEE Conference on Industrial Electronics and Applications, pp 2054$2057,2007$.

[11] S.H.Rasouli, A.Amirabadi, A.Seyedi and A.Afazalikusha, "Double Edge Triggered Feedback Flip-Flop in Sub 100nm Technology,” IEEE Conference, 2006.

[12] Wing-Shan Tam, Sik-Lam Siu, Chi-Wah Kok, and Hei Wong. "Double Edge-Triggered Half-Static Clock-Gated
D-Type Flip-Flop". IEEE International Conference of Electron Devices and Solid-State Circuits (EDSSC), 2010.

[13] Keisuke Inoue and Mineo Kaneko," Variable-Duty Cycle Scheduling in Double-Edge-Triggered Flip-FlopBased High-Level Synthesis,” IEEE Conference, 2011.

[14] Fatemeh Aezinia, Sara Najafzadeh, and Ali AfzaliKusha, "Novel High Speed and Low Power Single and Double Edge-Triggered Flip-Flops," IEEE Conference 2006.

[15] Hossein Karimiyan Alidash, Sayed Masoud Sayedi and Hossein Saidi, "Low-Power State-Retention Dual EdgeTriggered Pulsed Latch," Proceedings of ICEE 2010, May 11-13, IEEE 2010.

[16] M. Pedram, "Power minimization in IC Design: Principles and applications," ACM Transactions on Design Automation of Electronic Systems, vol. 1, pp.356, Jan. 1996

[17] G. E. Tellez, A. Farrahi, and M. Sarafzadeh, "ActivityDriven Clock Design for Low Power Circuits," Proceedings of the IEEWACM International Conference on Computer-Aided Design (ICCAD), pp. 62-65, 1995 